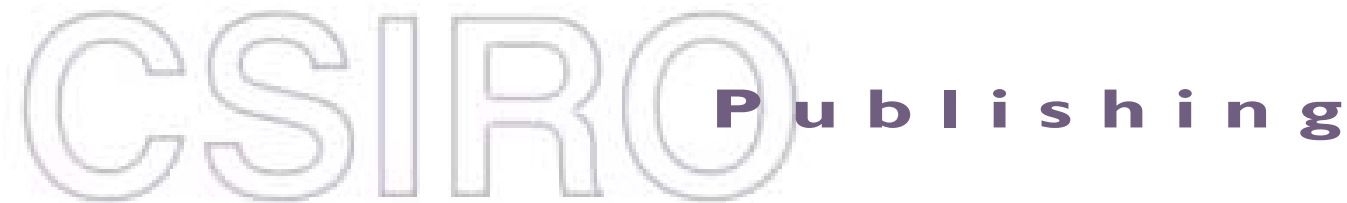

\section{Publications of the Astronomical Society of Australia}

Volume 19, 2002

(C) Astronomical Society of Australia 2002

An international journal of astronomy and astrophysics

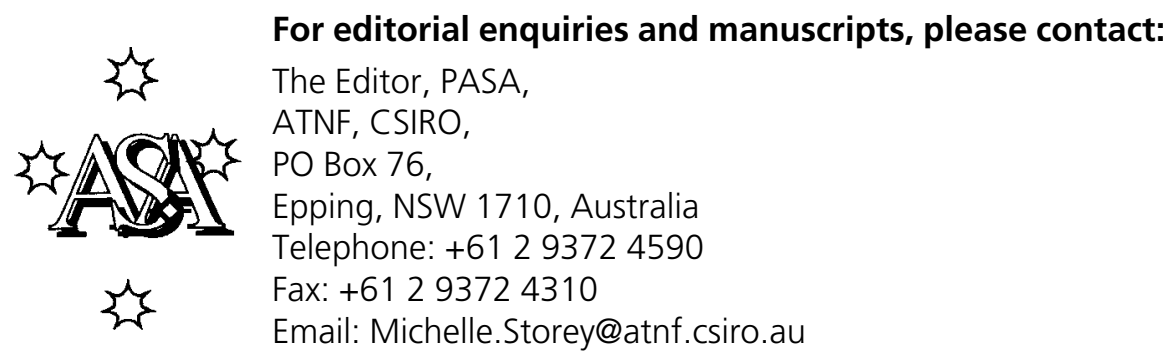

For general enquiries and subscriptions, please contact: CSIRO Publishing PO Box 1139 (150 Oxford St)

Collingwood, Vic. 3066, Australia

Telephone: +6139662 7666

Fax: +61 396627555

Email: publishing.pasa@csiro.au

C S I RO

PUBLISHING Published by CSIRO Publishing

for the Astronomical Society of Australia

www.publish.csiro.au/journals/pasa 


\title{
The IRAIT Project
}

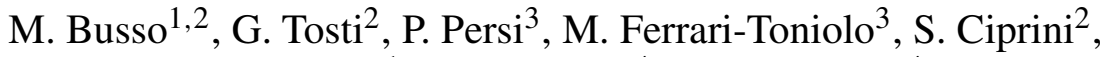 \\ L. Corcione ${ }^{1}$, F. Gasparoni ${ }^{4}$ and M. Dabalá ${ }^{4}$ \\ ${ }^{1}$ INAF — Osservatorio Astronomico di Torino, 10025 Pino Torinese, Italy \\ busso@to.astro.it, corcione@to.astro.it \\ ${ }^{2}$ Dipartimento di Fisica, Via A. Pascoli, 06123 Perugia, Italy \\ Gino.Tosti@ fisica.unipg.it, Stefano.Ciprini@pg.infn.it \\ ${ }^{3}$ IAS-CNR, via Fosso del Cavaliere, 00100 Roma \\ persi@ias.rm.cnr.it, ferrari@ias.rm.cnr.it \\ ${ }^{4}$ Tecnomare SpA, San Marco 3584, 30124 Venezia, Italy \\ gasparoni.f@tecnomare.it \\ dabala.m@tecnomare.it
}

Received 2001 August 1, accepted 2002 February 13

\begin{abstract}
We present the status of the project IRAIT (the Italian Robotic Antarctic Infrared Telescope) that will be hosted at Dome $\mathrm{C}$ in the Italo-French Concordia station. We review the main scientific motivations of the effort, and describe the characteristics of the telescope, which has been completed and is now under test at the Coloti-Montone site operated by the University of Perugia. Then we describe the design of the mid-IR camera that is under construction, showing some examples of the applications on the basis of the twin instrument TIRCAM II, now operating at the Italian Infrared Telescope TIRGO.
\end{abstract}

Keywords: infrared: general — telescopes — surveys — stars: formation — ISM: clouds: Magellanic Clouds — stars: AGB and post-AGB

\section{Introduction}

The Antarctica Plateau is the best place on the Earth to perform astronomical infrared observations in the 2-20 $\mu \mathrm{m}$ atmospheric windows and beyond, thanks to extremely low sky background emission, unique atmospheric transparency, and the possibility of passively cooling instrumentation to low temperatures.

The Dome C site, at $3200 \mathrm{~m}$ above sea level on the Antarctic Plateau, jointly exploited by Italian and French teams in the framework of the Concordia project, presents exceptionally cold and dry conditions. Site testing campaigns have been performed during the last years in summer time, and compared to other Antarctic sites (Valenziano 1995, 1999, Valenziano \& dall'Oglio 1999, Hidas et al. 2000). New data extended to winter time are expected in the period 2002-2003 from the Australian AASTO system (Storey 1998, Storey et al. 1999). Data collected so far points towards Dome $\mathrm{C}$ being the best place known on Earth for high-quality low sky background observations in the mid-infrared. Optimistic expectations on the sky conditions foresee that new windows may be opened in the spectral region between 20 and $40 \mu \mathrm{m}$, and that the 5-20 $\mu \mathrm{m}$ windows may be broader, more stable, and less affected by absorption and emission than in any other place. This is very important for long wavelengths, otherwise accessible from expensive, short lived space missions. Technology offers now the possibility of exploiting these opportunities with twodimensional detector arrays of doped semiconductors (e.g. $\mathrm{Si}$ : As and $\mathrm{Si}: \mathrm{Sb}$ ). Many open problems in star formation, late stages of stellar evolution, planetary physics, and extragalactic astronomy would obtain large benefits from an observatory in Dome $\mathrm{C}$, with access to those exotic windows.

Several difficulties must be overcome, and this is an important challenge for European technology. Indeed, first of all environmental conditions require robotic, remotely-controlled operations for the infrared telescope. Secondly, the above mentioned detectors require modern closed-circuit cryogenic techniques reaching very low temperatures (4-6K). Recent innovation both in detector technology itself, and in the cryogenic equipment for their use, has so far come mainly from outside Europe (USA, Japan). Exploiting a European facility for high-level infrared astronomy can promote European technology in the strategic fields of detectors and very low temperature systems.

We describe here the IRAIT project (Tosti et al. 1997), which is aimed at starting a permanent observatory in Dome C. It is based on an $80 \mathrm{~cm}$ telescope, and will be completed on the basis of a $4 \mathrm{yr}$ plan. The first two years (2002, 2003) will see the construction of the mid-infrared camera and the start of its integration with the telescope, which is already built and under test. The following period will then see the expedition and the start of operations in Antarctica. We intend this last phase as our participation in implementation of the Antarctic base of Dome C, and we are ready to offer IRAIT as a European facility. In order to minimise the difficulties we plan to use our telescope mainly in survey mode, to scan selected areas of the southern sky (see the next paragraph). We also want to use our facility as a test bench for larger projects, in particular 
for the joint international studies currently under way for larger telescopes, as in the Australian project of the Douglas Mawson Telescope (DMT: see e.g. Burton et al. 2000, Burton, Storey, \& Ashley 2001). In what follows we first recall the main scientific motivations for our efforts (Section 2), then describe the telescope (Section 3) and the design of the mid-infrared camera (Section 4).

\section{A Few Scientific Objectives}

After the IRAS, 2MASS, and DENIS surveys the whole sky has been covered at almost all wavelengths of the infrared range from 0.8 to $100 \mu \mathrm{m}$, except in the $\mathrm{L}^{\prime}$ $(3.8 \mu \mathrm{m})$ and $\mathrm{M}^{\prime}(4.8 \mu \mathrm{m})$ atmospheric windows. At longer wavelengths, however, the IRAS data suffer from extremely poor resolution, and surveys with higher resolving power are needed. Observations in the IRAS photometric bands at long wavelengths are extremely important for many astrophysical issues (see below). Although they can be performed from a conventional ground based 'infrared' site, they are severely hampered by the sky and instrumental background emission. For this reason, no large-scale surveys have been performed nor are even planned from the ground beyond the $2 \mu \mathrm{m}$ window.

Future space missions in this spectral range are sometimes primarily dedicated to pointed observations, like in the cases of SIRTF (Deutsch \& Bicay 2000) and NGST (Angel, Burge, \& Wool 1998). Otherwise they are aimed at surveys of limited areas (IRIS: see e.g. Onaka 2000). Missions fully dedicated to wide field surveys such as NGSS, a four colour all sky survey (Seguel et al. 2000), or PRIME, a 0.8-3 $\mu \mathrm{m}$ all sky survey (Zheng et al. 2000), are not yet approved.

As a first application of our Antarctic telescope we shall therefore discuss projects that adopt a survey mode of operation. We shall in particular discuss surveys on limited areas in selected fields of stellar evolution studies (e.g. initial and late phases of high and low mass star evolution). For reasons of space we are forced to skip other relevant fields, like studies of the surface composition of asteroids from their mid-IR colours, and extragalactic research, e.g. that devoted to star-bursts in nearby galaxies, which are well within the reach of an $80 \mathrm{~cm}$ telescope in Antarctica. One has also to take into account that large telescopes (Keck, Gemini, VLT, NGST) are (or will be) equipped with very sensitive imaging, or spectroscopic instruments working in the 3-20 $\mu \mathrm{m}$ range. Some will soon have interferometric capabilities in the same spectral range (e.g. VLTI, LBTI). There is therefore a strong need for establishing exhaustive catalogues of relatively faint sources and atlases in the 3-20 $\mu \mathrm{m}$ range for the preparation and calibration of the observing programmes to be conducted with these instruments.

Dedicated telescopes are currently being designed for the wavelengths near 1-2 $\mu \mathrm{m}$ (e.g. VST, VISTA, etc.), to provide future targets for telescopes of the $10 \mathrm{~m}$ class. A similar effort should be done beyond $2 \mu \mathrm{m}$, and we believe we can provide it.
In general, many exciting scientific targets easily come to an astronomer's mind, when thinking of an infrared observatory in Antarctica, but obviously only a limited part of them can be achieved with an $80 \mathrm{~cm}$ telescope. Considering the excellent transparency of the atmosphere and the low temperature of the site, we are performing a series of checks and upgrades to our instrumentation, as suggested by a specialised consulting engineering company (Tecnomare, Venice, Italy), to ensure that the telescope and camera emissivity are kept low enough to exploit the benefits of the Antarctic site. According to the models we presently have of the final outcome of these improvements, we estimate a transmission efficiency of the whole system near 0.7; the quantum efficiency of the detector is near 0.8 ; for a PFOV of 3 arcsec/pixel and a very favourable temperature condition $\left(-50^{\circ} \mathrm{C}\right)$, we can derive a background noise of $2.7 \mathrm{mJy}$ in $1 \mathrm{~h}$ of integration at $10 \mu \mathrm{m}$; at $20 \mu \mathrm{m}$ this number should be doubled. A more realistic average performance, for winter operations, may be worse than the given estimates by a factor of two, but this still involves exceptionally small background noise levels, as compared to what can be normally obtained in good astronomical sites with a small telescope. With these possibilities in mind, we are confident that even a moderate size telescope like ours will provide significant results in many fields, some of which are reviewed here.

\subsection{Interstellar Medium and Star Formation}

One of the main objectives of infrared observations at Dome $\mathrm{C}$ is to perform a deep and large-scale survey between 3 and $20 \mu \mathrm{m}$ of Southern Hemisphere star forming regions. This will allow a search for very young stellar objects (YSOs) and a correct census of the young stellar population in giant molecular clouds (GMCs) and dark clouds (DCs). A study of their initial mass function (IMF) will be possible from these observations. We propose three subjects for the mid-IR survey of star forming regions:

\section{a) Southern Dark Clouds}

Dark clouds are defined as regions of the sky where apparent surface densities of stars are reduced compared to surrounding environments. Lynds (1962) published a catalogue of 1802 dark clouds north of $\delta=-33^{\circ}$, obtained from a visual inspection of the Palomar Sky Survey Plates. For many years the DCs reported in this catalogue were objects of extensive observations from the infrared to the radio region of the spectrum. The main conclusions of these observations are that DCs represent ideal laboratories for the study of low mass star formation.

A complement to this catalogue for Southern Hemisphere DCs has been obtained by Hartley et al. (1986). They found 1101 new dark clouds with $\delta \leq-33^{\circ}$, in an area of the sky of $\sim 15$ square degrees. Only about $16 \%$ of these new DCs contain embedded IRAS sources, and 169 have been identified as dense small cometary cores (Bok globules). Subsequent near IR studies of a selected number of Southern Hemisphere DCs (Persi et al. 1990; Santos 
et al. 1998) with IRAS counterparts have shown that multiple systems of low mass stars are forming in these regions, and that near IR observations do not always discriminate YSOs from field stars. An homogeneous imaging survey, performed at 10 and $20 \mu \mathrm{m}$, is important to identify very easily the YSOs associated with the DCs, and to make statistical studies of low mass star formation. In addition, it will be possible to correlate the global characteristics of the DCs derived from the existing radio data, with the measured properties of the YSOs obtained from the analysis of their spectral energy distributions.

\section{b) The Chamaeleon Complex Molecular Cloud}

The Chamaeleon complex is one of the most interesting regions for studying very low mass star formation, given its proximity to the $\operatorname{Sun}(D \approx 160 \mathrm{pc})$ and its high galactic latitude $\left(b \sim 14^{\circ}-17^{\circ}\right)$. The complex is formed by six dark clouds (Cha I, Cha II, Cha III, DC302-16.9, G29517, and G298-13) extending for about 10 square degrees. Given its low declination $\left(\approx-78^{\circ}\right)$, the region is particularly suitable for Antarctic observations. Chamaeleon has been surveyed at large scale in $\mathrm{CO}$ lines (Boulanger et al. 1998) and in X-rays (Alcala et al. 1995). JHK images have been obtained in a small part of the complex, particularly in Cha I (Cambresy et al. 1998; Persi et al. 1999; Gomez $\&$ Kenyon 2001), while images at 6.7 and $14.3 \mu \mathrm{m}$ have been taken with ISOCAM (the infrared camera on board the ISO satellite) in selected areas of Cha I, Cha II, and Cha III (Persi et al. 2000). These last observations have shown the importance of mid-IR studies of these regions in detecting very low mass YSOs at the limit of hydrogen burning. New young brown dwarfs can be detected from large-scale mid-IR images as proposed by IRAIT in this complex molecular cloud.

\section{c) The Giant Molecular Cloud NGC 6334}

The giant molecular cloud/Hir region complex is unique among the star forming complexes in having at least seven distinct sites of massive star formation detected from radio and far IR observations. This Southern Hemisphere region $\left(\delta \sim-37^{\circ}\right)$ covers an area of about 2 square degrees, and is considered an ideal laboratory to study the effects of stellar UV radiation on the surrounding interstellar medium. Detailed infrared studies of NGC 6334 cover only a small part of the complex. The only large-scale infrared imaging survey was obtained with the SPIREX telescope at the South Pole in narrow- and broad-band filters at 3 and $4 \mu \mathrm{m}$ (Burton et al. 2000). Thermal dust imaging at 12 and $20 \mu \mathrm{m}$ was also obtained toward three centres of NGC 6334 (NGC 6334 F, A, and V) (Kraemer et al. 1999; Persi et al. 1998). All these observations have shown the importance that a more wide thermal survey of the complex is needed in understanding the processes of massive star formation.

\subsection{Late Stages of Stellar Evolution}

Low mass stars (LMS) and intermediate mass stars (IMS) in their late evolutionary stages populate the sequence in the H-R diagram known as the asymptotic giant branch
(AGB). Here they undergo radial pulsations in various modes, which trigger an intense mass loss, responsible for the creation of a circumstellar envelope of gas and dust (see e.g. Habing 1996). This envelope is often opaque to visible photons coming from the central star, due to dust grains condensing above the stellar extended atmosphere; it is nonetheless a source of thermal infrared radiation, especially in the range $2-40 \mu \mathrm{m}$ (see e.g. Busso et al. 1996).

For this reason, and for the optical properties of silicates, amorphous carbon, and silicon carbide ( $\mathrm{SiC})$, which are the main constituents of circumstellar grains, these near and mid-IR wavelengths are a preferred diagnostic tool to investigate the physical and chemical composition of dusty circumstellar environments around AGB stars (Willems \& de Jong 1986; van der Veen \& Habing 1988; Waters et al. 1996; Epchtein, Le Bertre, \& Lepine 1990; Guglielmo, Le Bertre, \& Epchtein 1997). The optical properties of AGB circumstellar environments derive primarily from the pulsational behaviour of the central variable star (either of Mira or of semi-regular type), which controls the mass loss mechanism. They are also strongly related to the chemical mix established in the stellar convective envelope, and in particular to the $\mathrm{C} / \mathrm{O}$ abundance ratio, which determines the type of dust grains that can be formed and is sensitive to many evolutionary parameters (Busso, Gallino, \& Wasserburg 1999).

A survey of AGB colours at long wavelengths can therefore contribute to the classification of red objects not seen in the visible, characterise their circumstellar envelopes, establish their mass loss rates, and study broad features from dust emission of different compounds. Another important result that can be obtained is the inventory of different AGB types in stellar systems of different properties. It is known, for example, that at low metallicities, like those of Magellanic Clouds or of the Galactic halo, the number ratio between carbon stars and $\mathrm{M}$ giants increases, but firm limits of mass within which the C-star phenomenon can occur at different metallicities are not known.

Such limits would be very useful for allowing an improvement in our knowledge of the final phases of stellar evolution and on the nucleosynthesis and chemical evolution in nearby galaxies, especially for elements like $\mathrm{C}, \mathrm{N}, \mathrm{O}$ and the neutron rich species belonging to the s-process, currently ascribed to AGB stars (Busso et al. 1999). This kind of knowledge can be obtained primarily from stellar systems of known distance modulus, like the Magellanic Clouds, because there the luminosities and, hence, the masses of AGB stars can be determined rather unambiguously (apart from local reddening processes). Searches for C-stars, Mira variables, post-AGB supergiants on their way to becoming white dwarfs, and for the planetary nebulae deriving from their envelope loss are key projects for our IR facility. Similar searches were attempted previously in the LMC and SMC; however, we would like to obtain a complete inventory, which is not possible from space-borne experiments of limited life, 


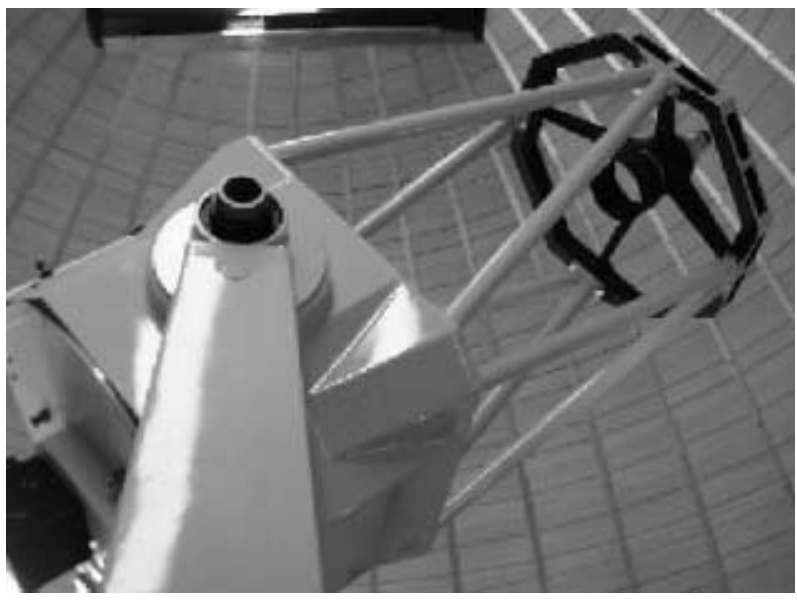

Figure 1 View of the IRAIT telescope at the Coloti observatory.

like ISO (the same will be true for future IR missions). The results can instead be reached today through surveys in near and mid-infrared wavelengths from Antarctica.

From the distance modulus of the LMC, the luminosities of AGB stars of different mass, and the fact that most of their flux is radiated at IR wavelengths, we can estimate the performance required by the observing instrument: this must reach sources as faint as 7-8 magnitudes (in the $5-10 \mu \mathrm{m}$ region). This is within the reach of a survey performed with a moderate size telescope, provided it is placed in a site like Dome C.

\section{The Telescope}

\subsection{Telescope Design, Mounting and Movements}

The telescope built for this research is a $\mathrm{f} / 20$ Cassegrainlike reflector, with a $0.8 \mathrm{~m}$ parabolic primary mirror and a wobbling secondary mirror suitable for the specific techniques of IR observations. It will be equipped with a mid-IR camera built in Italy, and possibly with a near IR camera built in France. The telescope is presently installed at an observational facility of the University of Perugia (see Figure 1), where we shall test it and the infrared cameras for both normal operation and remote control.

In order to minimise the telescope radiative emission, the optical system takes into account the size of the IR array. The secondary mirror is used as the entrance pupil so that the edge and the mount of the primary mirror are not visible from the focal plane. The optical characteristics of the telescope are listed in Table 1. The hole of the primary mirror is larger than strictly necessary, so that it will be possible to test the telescope with secondary mirrors having different focal ratios (the one mounted now is actually $\mathrm{f} / 10$ ). A cold stop is also included in the camera optics, as well as a cold demagnification optical system ahead of the detector, as described later. The telescope has rigid alt-azimuth mounting. We adopt this solution because the main support axes are always vertical and horizontal and the stresses on the axes hardly vary with the direction in which the telescope is pointing. Besides, while we are testing the telescope in Italy, it will operate
Table 1. The IRAIT main characteristics

\begin{tabular}{ll}
\hline Effective primary diameter & $800 \mathrm{~mm}$ \\
Clear aperture & $750 \mathrm{~mm}$ \\
Primary focal length & $2400 \mathrm{~mm}$ \\
Telescope focal length & $16000 \mathrm{~mm}$ \\
Linear obscuration ratio & 0.18 \\
Unvignetted field of view & $10 \mathrm{arcmin}$ \\
Back focal length & $800 \mathrm{~mm}$ \\
Cassegrain scale & $12.5^{\prime \prime} / \mathrm{mm}$ \\
\hline
\end{tabular}

in Antarctica, in an almost polar configuration and with minimal focal plane rotation. An alt-azimuth mounting is therefore more practical. All movements of the telescope are through worm and worm-wheel drivers on the altitude and azimuth axes. We used a 360 teeth worm-wheel having a diameter of $640 \mathrm{~mm}$ for the altitude axis and a 360 teeth worm-wheel having a diameter of $740 \mathrm{~mm}$ for the azimuth axis. The worms were coupled to the motor shafts without any further gear reduction stages, thus the value of the total gear ratio is $360: 1$. The position of the axes is revealed by incremental encoders with impulse of zero (18000 pulses/rev which yield a total resolution of $0^{\prime \prime} .05$ on sky for both axes). The maximum velocity of the movement is 1.5 degrees s $\mathrm{s}^{-1}$ and the telescope is limited to observations of objects that are at least 15 degrees above the horizon. The telescope is limited to complete azimuth rotations of not more than 270 degrees in the same direction to avoid problems with the cables. We used DC brushless motors for both the axes. The main advantages offered by this type of motor are: high power/weight ratio; high continuous accelerations; low inertia; excellent reliability; reduced sizes; notable capability of overload; minimal maintenance; rotation uniform also in the regime of low velocity; high efficiency; high velocity; maximum coupling to almost all the velocities; low level of noisiness; small generation of heat.

\subsection{Telescope Control, Auxiliary Equipment and Software}

The motor controllers we used are optimised to work in systems of distributed control in which more than one axis must be checked independently; they manage the position, speed, and current loops. The power stage of each motor is integrated in the controller. The controllers are AC powered $(18-30 \mathrm{~V})$ and the motors were designed to operate at $24 \mathrm{~V}$. Each controller is linked to the computerised telescope control system by a RS-232 serial line.

A dedicated software module was developed to manage the controller's instruction set and the communication protocol. All the fast and time-critical functions necessary to control the servo-feedback loop during slewing, tracking, and guiding of the telescope are provided by dedicated programs written using the controller high-level programming language.

Other necessary devices that are integrated into the system are a GPS receiver for time synchronisation, a conditioning system to protect all the computers and 


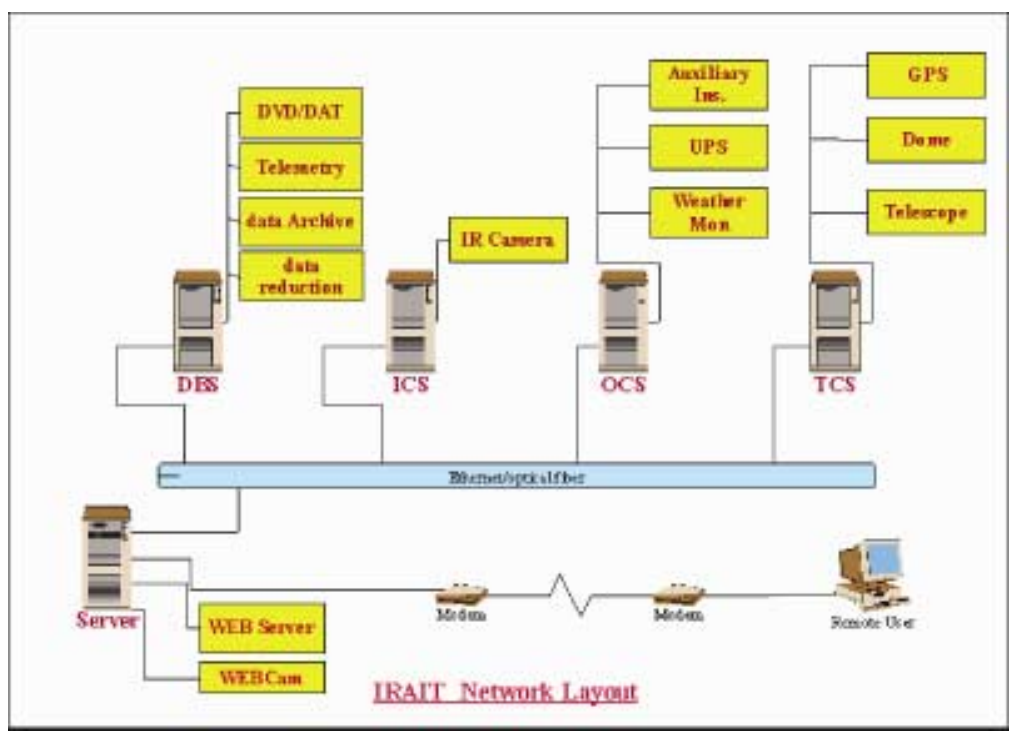

Figure 2 The IRAIT network.

electronic systems, and a weather monitor system (temperature, pressure, wind velocity and direction, humidity, snow, clouds). A dome to protect the telescope has been designed and two solutions have been selected: either an insulated double container with a fold-off canopy over it where the telescope can remain unshielded from the sides, or a true, more conventional dome. The final choice is pending.

The control system of the telescope (Figure 2) is under development in collaboration with the Technology Commission of Programma Nazionale delle Ricerche in Antartide (PNRA), which is responsible for the development of a centralised and coordinated management of all the scientific experiments supported by PNRA in Italy.

In order to define a control system architecture making the implementation of the software easier, we followed the prescription of the NASA/NBS Standard Reference Model for Telerobot Control System Architecture. It has been designed to operate both from the Antarctica base and, occasionally, from Europe. Remotely, it will be possible to prepare, modify and upgrade the scheduling, use the data obtained by the IR camera, control the progress of the schedule, monitor the system with full access to all the tasks, recover system alarms, interrupt and reboot the system, upgrade the system software, backup the system parameters and scientific data, and test the optical, mechanical and electronic parts of the system. The object oriented control software was designed to run under Windows NT 4.0 operating system and is written in C, $\mathrm{C}++$. In order to test different hardware solutions for the final design of the Antarctic telescope, we introduced a Telescope Control Macro Language (TCML). TCML is a script language which includes commands to perform all the tasks needed to operate in an automatic way an astronomical telescope. It derives from the past experience we have in building robotic telescopes (Tosti, Pascolini, \& Fiorucci 1995).

\section{The Italian mid-IR Camera}

The camera is designed to operate with the Boeing (exRockwell) high-flux Si : As or Si : Sb $128 \times 128$-pixel focal plane arrays (FPA) for ground based, high background observations. The pixel size is $75 \mu \mathrm{m}$. These detectors can operate with high quantum efficiency in the range 5-27 $\mu \mathrm{m}$ (Si : As) or 5-40 $\mu \mathrm{m}$ (Si: Sb). So far we have acquired experience in operating with such devices using the He-cooled dewar and the acquisition electronics of the TIRCAM (TIRgo InfraRed CAMera) camera (Persi et al. 1994) in its latest upgrade (TIRCAM II, see Persi et al. 2001) developed by the groups in the Institute of Space Astrophysics (IAS) and in the Astronomical Observatory of Torino (OATO). In the final configuration the cooling will be assured by a closed circuit cryogenic system working at a temperature of about $6 \mathrm{~K}$.

The camera will be equipped with a demagnification optical system designed to obtain the best compromise between spatial resolution and optimal sampling of the source images: during the test period the camera will be optimised to observe at $10 \mu \mathrm{m}$. In the first phase we plan to use the $\mathrm{Si}$ : As array, so here we outline the methods through which we will achieve our goal, divided according to the various sub-systems of the camera/telescope unit needed to operate such an array.

The front-end electronics are the first circuit for signal handling before passing the video signals coming from the detector to the acquisition system (16 in all) for A/D conversion and for further digital processing. In the particular case in which the $\mathrm{Si}$ : As detector is used, we need a 16 channel pre-amplifier with a bandwidth up to $3 \mathrm{MHz}$. In addition, we foresee a circuit section for biasing the detector and for buffering the control signals for the readout procedure. The acquisition system consists of a small rack housing some processing units based on digital signal processors (DSP). The control signals (digital clocks for detector scanning and triggers for the conversion units) 


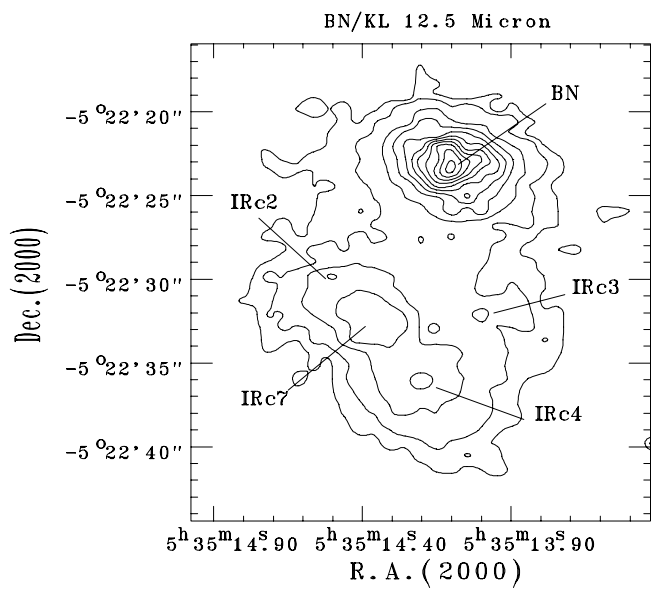

Figure 3 The BN-KN nebulae in Orion, as observed by TIRCAM II, a camera which is photometrically the twin of the IR camera for the IRAIT project.

are supplied by a sequencer based on a DSP. Data transfer is guaranteed at $10 \mathrm{Mbits}$ up to $1 \mathrm{~km}$ distance. A different link with the same electrical characteristics is devoted to command handling between remote host and sequencer. A processing personal computer unit, hosted in an industrial standard insulated rack-like crate, covers all the needs of digital processing (e.g. co-adding) on data collected by the A/D units, as requested by the different integrating modes that an infrared observation may need. Six 3-channel 14 bits $3 \mathrm{MHz}$ A/D converter units are available for parallel analogue to digital conversion on the 16 channels: each unit has a local processor controlling 3 A/D channels, storing data onto a 16 Mbyte memory unit on board and handling the communications with the controlling host. This gives us a total of 18 channels. Of them 16 are needed, and two are used as backups. The acquisition electronics will be placed in a box directly on the dewar. The acquisition modules are industrial standards similar to those used for the TIRCAM II project (Persi et al. 2001).

The photometric system will include standard filters in the mid-IR (the $\mathrm{N}$ and Q filters, plus filters at 8.7, 9.8, $10.3,11.2$, and $12.4 \mu \mathrm{m}$, each $1 \mu \mathrm{m}$ wide, and a circular variable filter (CVF) for spectrophotometry). Such a simple scheme is already capable of high performance in studying the main features of reddened sources. In Figure 3 we present, as an example, an observation of the BN-KL region of the Orion star forming region complex, made by TIRCAM II at the TIRGO infrared telescope.

\section{Future Developments}

In order to make the telescope fully compatible with the Dome C climatic condition we shall now implement a series of upgrades to the existing telescope (optimising thermal characteristics, insulating exposed parts, etc.); we shall also finish and interface the IR camera. In the meantime we have pursued the creation of an international network for exploiting IRAIT also as a test bench for future, larger projects.
As a result, researchers from the Observatory of Nice (N. Epchtein) and from the UNSW (J. Storey et al.) have been particularly active in providing us with suggestions and help. There is a plan for transforming IRAIT into an Italo-French telescope at $50 \%$ share, according to which a second camera, for shorter wavelengths $(1.2-5 \mu \mathrm{m})$ might be provided by a French project. We thus hope that our relatively small instrument can become one of the starting points for building a modern international observatory at Dome C.

\section{Acknowledgments}

Many ideas and scientific objectives for IRAIT have been suggested by N. Epchtein, who has become a stable reference point and a collaborator in the deepest sense: we gratefully acknowledge here his invaluable help. Our thanks go also to John Storey for his advice on specific solutions for Antarctica, going as far as to spend a dedicated review visit to IRAIT near Perugia in April 2001. We are grateful to the organisers of the Third International Conference on Astrophysics at Dome C in Antarctica for the very stimulating occasion and for the many collaborative efforts that have been started in Hobart. This research is funded by the Italian Progetto Nazionale per le Ricerche in Antartide.

\section{References}

Alcala, J. M., Krautter, J., Schmitt, J. H. M. M., Covino, E., Wichmann, R., \& Mundt, R. 1995, A\&AS, 114, 109

Angel, J. R. P., Burge, J. H., \& Wool, N. J. 1998, SPIE, 3356, 185

Boulanger, F., Bronfman, L., Dame, T. M., \& Thaddeus, P. 1998, A\&A, 332, 273

Burton, M. G., et al. 2000, ApJ, 542, 359

Burton, M. G., Storey, J. W. V., \& Ashley, M. C. B. 2001, PASA, 18, 158

Busso, M., et al. 1996, A\&A, 311, 253

Busso, M., Gallino, R., \& Wasserburg, G. J. 1999, ARA\&A, 37, 239

Cambresy, L., Copet, E., Epchtein, E., de Batz, B., Borsenberger, J., Fouque, P., Kimeswenger, S., \& Tiphene, D. 1998, A\&A, 338,977

Deutsch, M.-J., \& Bicay, M. D. 2000, Ap\&SS, 273, 187

Epchtein, N., Le Bertre, T., \& Lepine, J. R. D. 1990, A\&A, 227, 82

Gomez, M., \& Kenyon, S. J. 2001, AJ, 121, 974

Guglielmo, F., Le Bertre, T., \& Epchtein, N. 1997, A\&A, 334, 609

Habing, H. J. 1996, A\&AR, 7, 97

Hartley, M., Tritton, S. B., Manchester, R. N., Smith, R. M., \& Goss, W. M. 1986, A\&AS, 63, 27

Hidas, M. G., Burton, M. G., Chamberlain, M. A., \& Storey, J. W. V. 2000, PASA, 17, 260

Kraemer, K. E., Deutsch, L. K., Jackson, J. M., Hora, J. L., Fazio, G. G., Hoffmann, W. F., \& Dayal, A. 1999, ApJ, 516,817

Lynds, B. T. 1962, ApJS, 7, 1

Onaka, T. 2000, ESASP, 456, 361

Persi, P., et al. 1994, Exper. Astron., 5, 363

Persi, P., Ferrari-Toniolo, M., Busso, M., Origlia, L., Robberto, M., Scaltriti, F., \& Silvestro, G. 1990, AJ, 99, 303

Persi, P., Tapia, M., Felli, M., Lagage, P. O., \& Ferrari-Toniolo, M. 1998, A\&A, 336, 1024

Persi, P., Marenzi, A. R., Kaas, A. A., Olofsson, G., Nordh, L., \& Roth, M. 1999, AJ, 117, 439

Persi, P., et al. 2000, A\&A, 357, 219 
Persi, P., Busso, M., Corcione, L., Marenzi, A., \& Ferrari-Toniolo, M. 2001, in IAS Internal Report, No. 6

Santos, N. C., Yun, J. L., Santos, C. A., \& Marrerios, R. G. 1998, AJ, 116, 1376

Seguel, J. C., et al. 2000, AAS, 197, 8113

Storey, J. W. V. 1998, in 192nd Meeting of AAS, 6227

Storey, J. W. V., et al. 1999, PASP, 111, 765

Tosti, G., Pascolini, S., \& Fiorucci, M. 1995, PASP, 108, 706

Tosti, G., et al. 1997, Astron. \& Astrop. Tran., 13, 67
Valenziano, L. 1995, Exper. Astron., 6, 83

Valenziano, L. 1999, in Astronomy and Astrophysics at Sub-mm Wavelengths, SIF1999, 95

Valenziano, L., \& dall'Oglio, G. 1999, PASA, 16, 167

van der Veen, W. E. C. J., \& Habing, H. J. 1988, A\&A, 194, 125

Waters, L. B. F. M., et al. 1996, A\&A, 315, L361

Willems, F. L., \& de Jong, T. 1986, ApJ, 309, L39

Zheng, W., et al. 2000, AAS, 197, 9306 